\title{
Hybrid Structural Reliability Analysis under Multisource Uncertainties Based on Universal Grey Numbers
}

\author{
Xingfa Yang, ${ }^{1,2}$ Jie Liu, ${ }^{1,3}$ Xiaoyue Chen, ${ }^{2}$ Qixiang Qing, ${ }^{2}$ and Guilin Wen $\mathbb{D}^{1,2,3}$ \\ ${ }^{1}$ State Key Laboratory of Advanced Design and Manufacturing for Vehicle Body, Hunan University, Changsha, Hunan 410082, China \\ ${ }^{2}$ Key Laboratory of Advanced Design and Simulation Techniques for Special Equipment, Ministry of Education, \\ Hunan University, Changsha, Hunan 410082, China \\ ${ }^{3}$ Center for Research on Leading Technology of Special Equipment, School of Mechanical and Electric Engineering, \\ Guangzhou University, Guangzhou 510006, China
}

Correspondence should be addressed to Guilin Wen; glwen@hnu.edu.cn

Received 26 December 2017; Accepted 8 March 2018; Published 17 April 2018

Academic Editor: Mohammad A. Hariri-Ardebili

Copyright (C) 2018 Xingfa Yang et al. This is an open access article distributed under the Creative Commons Attribution License, which permits unrestricted use, distribution, and reproduction in any medium, provided the original work is properly cited.

Nondeterministic parameters of certain distribution are employed to model structural uncertainties, which are usually assumed as stochastic factors. However, model parameters may not be precisely represented due to some factors in engineering practices, such as lack of sufficient data, data with fuzziness, and unknown-but-bounded conditions. To this end, interval and fuzzy parameters are implemented and an efficient approach to structural reliability analysis with random-interval-fuzzy hybrid parameters is proposed in this study. Fuzzy parameters are first converted to equivalent random ones based on the equal entropy principle. $3 \sigma$ criterion is then employed to transform the equivalent random and the original random parameters to interval variables. In doing this, the hybrid reliability problem is transformed into the one only with interval variables, in other words, nonprobabilistic reliability analysis problem. Nevertheless, the problem of interval extension existed in interval arithmetic, especially for the nonlinear systems. Therefore, universal grey mathematics, which can tackle the issue of interval extension, is employed to solve the nonprobabilistic reliability analysis problem. The results show that the proposed method can obtain more conservative results of the hybrid structural reliability.

\section{Introduction}

In most cases, uncertain structural parameters could have undesired effects on the reliability of structures and systems. Normally, probability model $[1,2]$ and fuzzy model [3] are utilized to obtain structural reliability based on probability theory and fuzzy set theory, respectively. Since probability and fuzzy models have been developed rapidly over the past decades, both of them have been applied in an ocean of engineering fields [4]. Unfortunately, when it comes to the situation that the experimental samples and data are not sufficient to construct the precise distribution or membership function, these two methods are no longer reliable.

Ben-Haim [5, 6] and Elishakoff [7] pioneered to develop the nonprobabilistic convex model to deal with the uncertainty lacking of information and introduced the concept of nonprobabilistic reliability. They suggested that uncertain parameters should be treated as convex set, such as interval and ellipsoid, so that uncertainty model can be obtained with the information of those parameters' bounds. Since then interval reliability analysis has arisen wide concern. Du [8] and $\mathrm{Du}$ et al. [9] systematically studied the structural reliability analysis consisting of interval parameters. Various techniques have also proposed to solve this kind of reliability and design problem [10-18]. However, there could be the circumstance that the designing parameters of a structure have different kinds of uncertainties. For example, the geometry parameters of the structure are random variable, but the subjected force is fuzzy [19], or vice verse [20]. Developing an effective method to solve this problem is vital for engineers to predict the structural reliability precisely. Haldar and Reddy [21] proposed two approaches to estimate the reliability of existing structures by considering both the randomness and the fuzzy uncertainty in some parameters. Ni and Qiu [22] 
presented an innovative method to calculate the structural reliability of linear and nonlinear structural systems when probabilistic, fuzzy, and nonprobabilistic uncertainties are involved. A multitude of researchers have worked this filed recently and fruitful achievements have been obtained [23-28], including calculating the reliability of motion mechanism, solving time-dependent problems, and designing metamaterials.

However, interval expansion is unavoidable when interval mathematics is used, which is often neglected by the researchers. Rao and Berke [29] proposed an interval-truncation approach to narrow interval expansion, but the truncation principle is hard to control. Shao and $\mathrm{Su}$ [30] applied interval finite element method to narrow interval expansion. In this study, we attempt to put forward alternative approach to handle the interval expansion problem in structural reliability analysis when hybrid uncertainties are involved. The main focus of this work will introduce a new approach by utilizing universal grey number to avoid interval expansion in reliability analysis. Meanwhile, techniques, like $3 \sigma$ criterion and equal entropy principle, are used as well.

The rest of this paper is organized as follows. Section 2 introduces an interval reliability model; Section 3 presents reliability analysis with random, fuzzy, and interval parameters and introduces the methods and theory of transformation among random, fuzzy, and interval variables. Section 4 introduces the universal grey number. Section 5 discusses and analyzes some numerical examples; the conclusion is given in Section 6.

\section{Interval Reliability Model}

For interval reliability analysis, structural performance is commonly expressed by performance function which is determined by failure criteria. Assuming that $\mathbf{Z}^{\mathbf{I}}$ is a $k$-dimension vector of interval variable with independent elements

$$
\begin{aligned}
\mathbf{Z} \in \mathbf{Z}^{\mathbf{I}} & =[\underline{\mathbf{Z}}, \overline{\mathbf{Z}}], \\
Z_{i} \in Z_{i}^{I} & =\left[\underline{Z_{i}}, \overline{Z_{i}}\right], \quad i=1,2, \ldots, k,
\end{aligned}
$$

where $Z_{i}$ and $\overline{Z_{i}}$ represent the lower and upper bounds of interval, respectively. Then the structural performance function can be expressed as

$$
Q=g\left(\mathbf{Z}^{\mathrm{I}}\right)=g\left(Z_{1}^{I}, Z_{2}^{I}, \ldots, Z_{k}^{I}\right) .
$$

The reliability can be defined as

$$
P_{r}=P\left\{g\left(\mathbf{Z}^{\mathbf{I}}\right) \geq 0\right\} .
$$

And limit-state function of the structure writes

$$
Q=g\left(\mathbf{Z}^{\mathrm{I}}\right)=g\left(Z_{1}^{I}, Z_{2}^{I}, \ldots, Z_{k}^{I}\right)=0
$$

which defines the limit-state surface. The basic variable space will be divided into safe region and failure region by the limitstate function, and if taking the example of two-dimensional linear performance function $Q=Z_{2}-Z_{1},\left(Z_{2} \in Z_{2}^{I}=\right.$ $\left.\left[\underline{Z_{2}}, \overline{Z_{2}}\right], Z_{1} \in Z_{1}^{I}=\left[\underline{Z_{1}}, \overline{Z_{1}}\right]\right)$, the safe region and failure

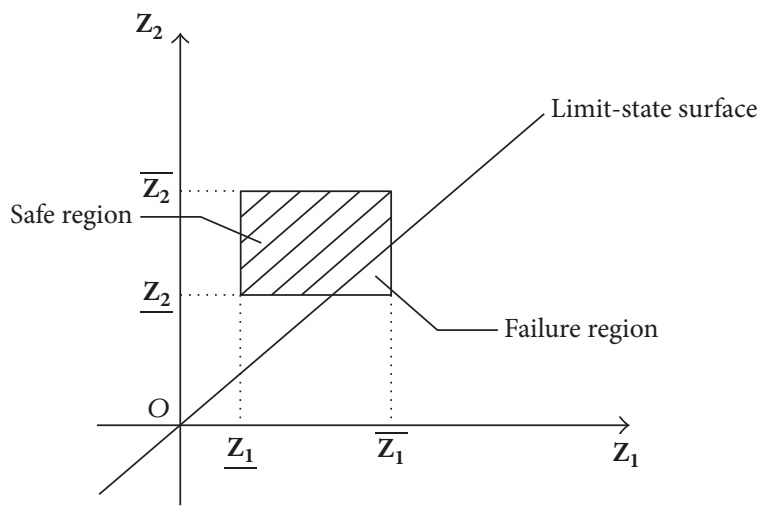

FIGURE 1: Scheme for safe region and failure region.

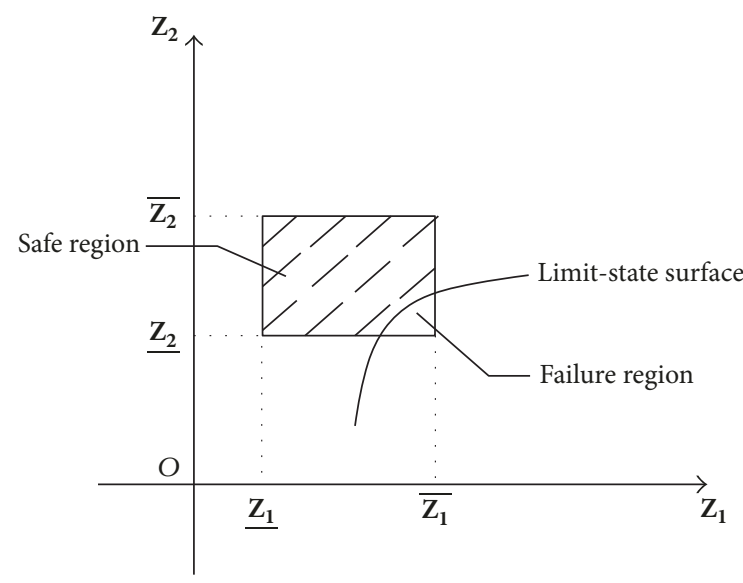

FIgURE 2: Nonlinear structural performance function.

region would be separated as shown in Figure 1. The interval safety probability can be defined as the ratio of safe region to the total area of variable space [22].

$$
P_{r}=\frac{V_{\text {safe }}}{V_{\text {total }}} .
$$

When the performance function is nonlinear or multidimensional, the variable space will be divided into safe region and failure region by limit-state surface or hyper-surface as well, as shown in Figure 2. So the safe probability can still be defined by the ratio of safe region to total variable region like (5).

\section{Reliability Analysis with Random, Fuzzy, and Interval Parameters}

Limit-state function with random variables, fuzzy variables, and interval variables can be expressed as follows:

$$
\begin{aligned}
Q & =g\left(\boldsymbol{x}, \widetilde{\mathbf{y}}, \mathbf{z}^{I}\right) \\
& =g\left(x_{1}, x_{2}, \ldots, x_{m}, \tilde{y}_{1}, \tilde{y}_{2}, \ldots, \widetilde{y}_{n}, z_{1}^{I}, z_{2}^{I}, \ldots, z_{l}^{I}\right) \\
& =0,
\end{aligned}
$$


where $\boldsymbol{x}=\left(x_{1}, x_{2}, \ldots, x_{m}\right)$ denotes a random variable vector with independent elements of normal distribution. The probability density function of $x_{i}(i=1,2, \ldots, m)$, which are the random variables, is $f_{i}\left(x_{i}\right)(i=1,2, \ldots, m) . \widetilde{\mathbf{y}}=$ $\left(\tilde{y}_{1}, \tilde{y}_{2}, \ldots, \tilde{y}_{n}\right)$ denotes a fuzzy variable vector with independent elements. Membership functions of $\tilde{y}_{j}(j=1,2, \ldots, n)$, the fuzzy variables, are represented by $\mu_{j}\left(y_{j}\right)(j=1,2, \ldots, n)$. $\mathbf{z}^{I}=\left(z_{1}^{I}, z_{2}^{I}, \ldots, z_{l}^{I}\right)$ denotes an independent nonprobabilistic interval variable vector, and $z_{k}^{I}=\left[z_{k}, \overline{z_{k}}\right](k=1,2, \ldots, l)$. So the structural hybrid reliability can be expressed as

$$
P_{r}=P\left(Q=g\left(\boldsymbol{x}, \widetilde{\mathbf{y}}, \mathbf{z}^{I}\right) \geq 0\right) .
$$

3.1. Transforming Fuzzy Variable into Random Variable. Entropy [31], a basic concept in thermodynamics, is used for expressing the degree of the irregular movement of molecules. It can be regarded as a measure of uncertainty. Entropy is not only a measure of random uncertainty but also fuzzy uncertainty. The measure of random uncertainty and fuzzy uncertainty are defined as probabilistic entropy and fuzzy entropy, respectively.

The probabilistic $H$ entropy of continuous variables $X$ is defined as follows:

$$
H_{X}=-\int_{R} f(x) \ln f(x) d x,
$$

where $f(x)$ is the probability density function of $X$.

The definition of fuzzy entropy can be described as:

$$
G_{Y}^{\prime}=-\int_{R} f^{\prime}(y) \ln f^{\prime}(y) d y,
$$

where $f^{\prime}(y)=\mu(y) / \int_{R} \mu(y) d y$ and $\mu(y)$ is the membership function of $Y$.

According to the principle that the equivalent probabilistic entropy equals to the fuzzy entropy, fuzzy uncertainty can be transformed into random ones. So the transformation from fuzzy entropy to equivalent random entropy can be constructed on the basis of (8) and (9):

$$
H=G^{\prime} \text {. }
$$

Supposing the equivalent random variable transformed from fuzzy variable $Y$ is $X$. The entropy of a normal variable can be obtained by evaluating (8) as

$$
H_{\mathrm{eq}}=-\int_{R} f(x) \ln f(x) d y=\ln (\sqrt{2 \pi e} \sigma) .
$$

The equivalent standard deviation of the transform from fuzzy variable to random variable can be obtained according to (11):

$$
\widehat{\sigma}=\frac{1}{\sqrt{2 \pi}} e^{G^{\prime}-0.5} .
$$

The equivalent mean is assumed to be the fuzzy variable mean and the correspondent mean value when the membership value is 1 , represented by $a$. Then the probabilistic density function of equivalent random variable with normal distribution can be obtained:

$$
\widehat{f}_{I}(x)=\frac{1}{\sqrt{2 \pi} \widehat{\sigma}} e^{-(1 / 2)((x-a) / \widehat{\sigma})^{2}} .
$$

3.2. Transforming Random Variable into Interval Variable. $3 \sigma$ principal reveals that most of the random variable values are in the interval of $(\mu-3 \sigma, \mu+3 \sigma)$ and the excess accounts for less than $0.3 \%$. So the equivalent probabilistic variable and probabilistic variable can be transformed into uncertain but limited interval variable.

$x$ is transformed into $x^{I}$, an interval variable located in $[\mu-3 \sigma, \mu+3 \sigma]$, where $\mu$ is the mean value of the normally distributed random variable and $\sigma$ is the deviation. The equivalent probabilistic uncertain variable of $\tilde{y}$, the fuzzy variable, is transformed into $\widetilde{y}^{I}$, the interval variable located in the interval of $[\widehat{\mu}-3 \widehat{\sigma}, \widehat{\mu}+3 \widehat{\sigma}]$. Then the hybrid structural reliability solving problem has turned into a nonprobabilistic reliability solving problem only with interval variables.

\section{Universal Grey Mathematics}

\subsection{Brief Introduction of Universal Grey Number}

Definition 1. Suppose discussed region $U=R$ (real number set). Then the universal grey number of $R$ is named universal grey number set, noted $g(R)$, and the element of $g(R)$ is named universal grey number, noted

$$
g=(x,[\underline{\mu}, \bar{\mu}]), \text { âNíl } x \in R, \underline{\mu}, \bar{\mu} \in R,
$$

where $x$ is the observation value, $[\mu, \bar{\mu}]$ is the grey information part, and $g^{(0)}=(0,[0,0])$ and $g^{(1)}=(1,[1,1])$ are the zero unit and unit, respectively. The universal grey number, whose observation part is naught but information part is not naught, is noted as $g^{\prime(0)}$ and named subzero unit. Zero unit and subzero unit are called by a joint name of universal zero unit, noted as $g^{\prime(0)}$.

Definition 2. $\forall g=(x,[\mu, \bar{\mu}]) \in g(R),-g=(-x,[\mu, \bar{\mu}])$ is called negative unit on $g$ in $g(R)$ and $g^{-1}=\left(x^{-1},\left[\underline{\mu}^{-1}, \bar{\mu}^{-1}\right]\right)$ is called inverse unit on $g$ in $g(R)$.

On the basis of Definitions 1 and 2, the four arithmetic operations of universal number are regulated. The multiplication operation of universal grey number meets closure, association law, and exchange law. The four arithmetic operations are as follows:

$$
\begin{aligned}
g_{1}+g_{2} & =\left(x_{1}+x_{2},\left[\frac{x_{1} \underline{\mu}_{1}+x_{2} \underline{\mu}_{2}}{x_{1}+x_{2}}, \frac{x_{1} \bar{\mu}_{1}+x_{2} \bar{\mu}_{2}}{x_{1}+x_{2}}\right]\right) \\
g_{1}-g_{2} & =\left(x_{1}-x_{2},\left[\frac{x_{1} \underline{\mu}_{1}-x_{2} \underline{\mu}_{2}}{x_{1}-x_{2}}, \frac{x_{1} \bar{\mu}_{1}-x_{2} \bar{\mu}_{2}}{x_{1}-x_{2}}\right]\right) \\
g_{1} \times g_{2} & =\left(x_{1} x_{2},\left[\underline{\mu_{1}} \underline{\mu}_{2}, \overline{\mu_{1}} \overline{\mu_{2}}\right]\right) \\
\frac{g_{1}}{g_{2}} & =\left(\frac{x_{1}}{x_{2}},\left[\frac{\underline{\mu}_{1}}{\mu_{2}}, \frac{\underline{\mu}_{1}}{\bar{\mu}_{2}}\right]\right), \quad\left(\text { if } g \neq g^{\prime(0)}\right) .
\end{aligned}
$$

4.2. The Transformation of Universal Grey Number and Interval Number. According to the definition of universal grey 
number, $\mu$ and $\bar{\mu}$ of $(x,[\underline{\mu}, \bar{\mu}])$, a universal grey number, can be, respectively, looked as the lowest and highest trust degree. So, when $\mu, \bar{\mu} \in[-1,1]$, a universal grey number can be expressed by an interval number, namely,

$$
(x,[\underline{\mu}, \bar{\mu}])=(\underline{\mu} x, \bar{\mu} x) .
$$

$\forall[a, b] \in I(R)$ (an interval number set) it can be expressed by an interval number $(x,[\underline{\mu}, \bar{\mu}])$ :

(1) When there is $a>0$, there is $[a, b]=(b,[a / b, 1])$.

(2) When there are $a b<0$ and $\max \{|a|,|b|\}=|a|$, there is $[a, b]=(a,[b / a, 1])$.

(3) When there are $a b<0$ and $\max \{|a|,|b|\}=|b|$, there is $[a, b]=(b,[a / b, 1])$.

(4) When there is $b<0$, there is $[a, b]=(a,[b / a, 1])$.

Universal grey number not only has the capability of interval analysis but also can avoid the disadvantage of interval computation, and effectively solve the issue of interval extension. Taking advantage of universal grey number, the interval number can be transformed into universal grey number when computing structural reliability, resulting in a more precise outcome.

Based on the discussion above, the proposed procedure for computing the hybrid reliability of the structural system and overcoming the interval expansion at the same time is briefly shown as follows:

(a) Input hybrid parameters, including random, interval, and fuzzy ones.

(b) Transform the fuzzy variable into equivalent random ones on the basis of the equal entropy principle.

(c) Convert the random and equivalent random variables to equivalent interval ones based on the $3 \sigma$ principal.

(d) Transform all interval variables into universal grey numbers based on (16).

(e) Calculate the hybrid reliability using (5) and (15).

\section{Numerical Example}

In this section, two classical numerical examples and one engineering problem are presented to manifest the validity of the proposed method.

5.1. A Cantilever Tube. A cantilever tube is taken as the first test example, which is presented in Figure 3. Both of the two forces on it are interval variables, and $F \in[2850,3150](\mathrm{N})$, $P \in[11700,12300](\mathrm{N})$. The thickness of the tube is denoted as $t$, which is fuzzy and its membership function is shown in Figure 4. Considering that, the stress at the right end of the tube should be less than $R_{y}$, which is a random variable and follows a normal distribution of $N(28,0.8)$. Then the limitstate function can be obtained as follows:

$$
g(\mathbf{X})=R_{y}-\sigma_{\max } .
$$

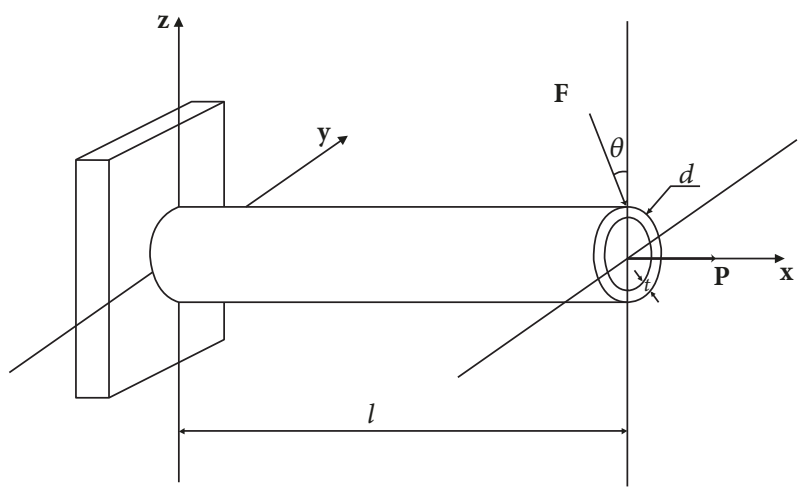

Figure 3: A cantilever tube.

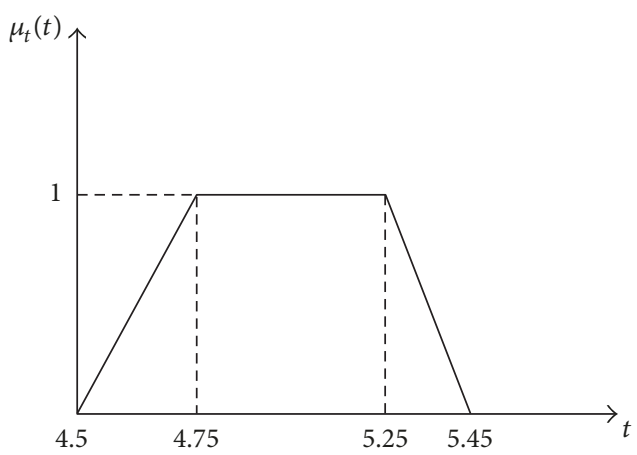

FIGURE 4: Membership function of $t$.

$\sigma_{\max }$ is calculated by the following equations:

$$
\begin{aligned}
\sigma_{\max } & =\frac{P+F \sin \theta}{A}+\frac{M}{I} \\
A & =\frac{\pi}{4}\left[d^{2}-(d-2 t)^{2}\right], \\
M & =F L \cos \theta \\
I & =\frac{\pi}{64}\left[d^{4}-(d-2 t)^{4}\right] .
\end{aligned}
$$

The reliability probability by using our approach is $90.47 \%$, which is a bit lower than the interval analysis result of $91.75 \%$. It is clearly shown that our approach can deal with the interval expansion problem such that our result is more conservative when compared with that obtained from interval analysis directly.

5.2. A Freely-Supported Beam. Figure 5 presents a freely supported beam subjecting to uniform load $q$, which is a random variable, and $q \sim N\left(210,3^{2}\right) \mathrm{kN} / \mathrm{mm}^{2}$. The values of section width $b$ and height $h$ are $120 \mathrm{~mm}$ and $240 \mathrm{~mm}$, respectively. Length $l$ of the beam is an interval variable, and $l \in l^{I}=[3880,4120] \mathrm{mm}$. The beam is made of 45 -steel and 


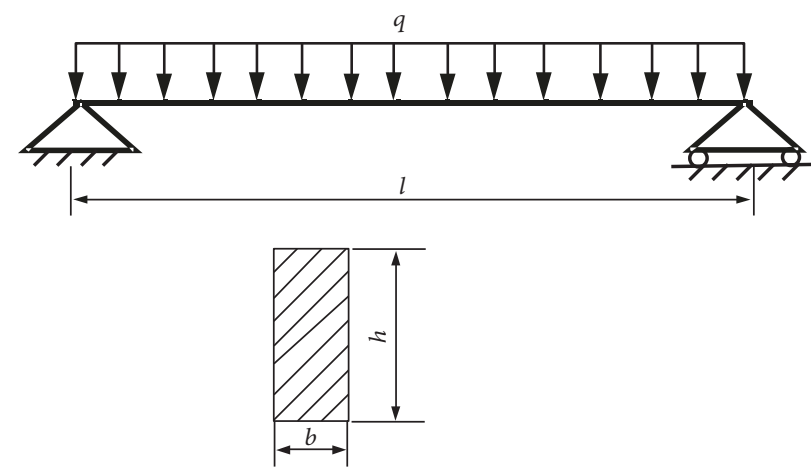

Figure 5: A freely supported beam.

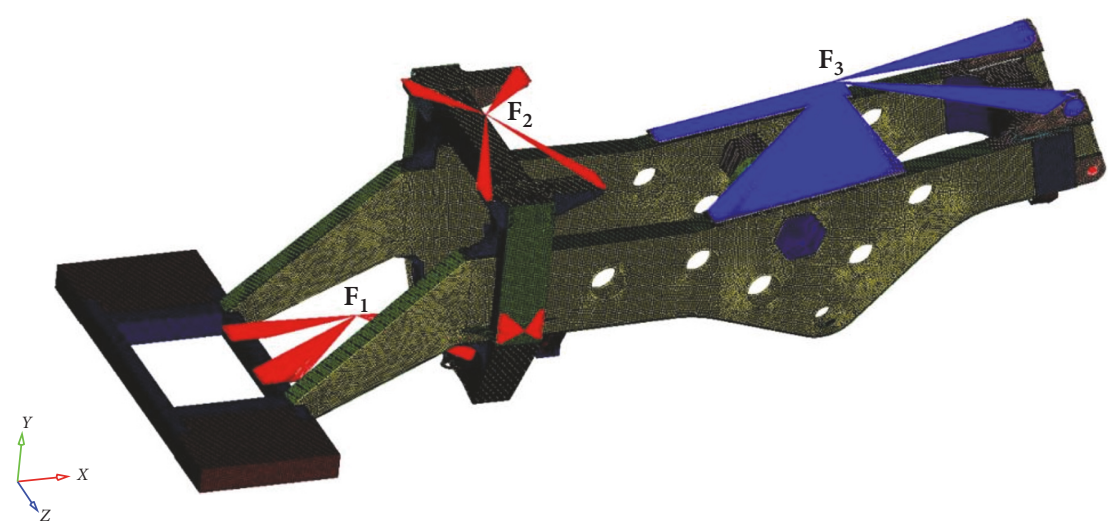

FIGURE 6: An automobile frame.

its strength $\widetilde{R}$ is a fuzzy parameter. The membership function of $\widetilde{R}$ is

$$
\mu_{R}(R) \begin{cases}0 & R<760 \\ \frac{R-720}{40} & 720 \leq R<760 \\ 1 & 760 \leq R<820 \\ \frac{900-R}{80} & 820 \leq R<900 \\ 0 & R>900 .\end{cases}
$$

Based on material mechanics, the maximum stress of the simple free beam is

$$
S=\frac{0.75 q l^{2}}{b h^{2}} .
$$

So the performance function can be denoted as

$$
Z=\widetilde{R}-\frac{0.75 q l^{2}}{b h^{2}}=0
$$

$\widetilde{R}$ is converted into equivalent normal distributed random variable, so the equivalent deviation is $\widehat{\sigma}=37.28$, and the equivalent mean $\widehat{\mu}=790$. Then the random variable $q$ and the equivalent random variable $R$ are transforming into interval variables of $q^{I}$ and $R^{I}$, respectively, and $q^{I} \in[201,219]$,
$R^{I} \in[679,901]$. Finally the the hybrid reliability is obtained and its value is 0.9009 , which is smaller than the outcome of interval analysis, 0.9687. Again, we can say that the proposed method can tackle the interval expansion problem and get more conservative reliability result.

5.3. An Automobile Frame. The proposed method is finally applied into calculating the reliability of a practical frame structure of a dump truck, which is shown in Figure 6. The frame is subjected to three major forces from engine assembly, carriage, and operation cabin. For the reason that these loads are alterable, they all have variation interval. The bounds of the interval parameters are presented in Table 1. Young's Modulus is a random variable because of the manufacturing error, and it follows a distribution of $E$ $N(2.1 e 5,50)$. The performance function is denoted as

$$
g(\mathbf{X})=d_{y}-d\left(E, F_{1}, F_{2}, F_{3}\right)
$$

where $d_{y}$ is the maximum vertical displacement allowed in the static state, and it is a fuzzy variable, whose membership function is

$$
\mu_{d_{y}}= \begin{cases}250\left(d_{y}-0.69\right) & 0.69 \leq d_{y} \leq 0.694 \\ 1 & 0.694 \leq d_{y} \leq 0.698 \\ 500\left(0.7-d_{y}\right) & 0.698 \leq d_{y} \leq 0.7\end{cases}
$$


TABLE 1: Bounds of interval loads to automobile frame.

\begin{tabular}{lcc}
\hline & Lower bound $(\mathrm{kN})$ & Upper bound $(\mathrm{kN})$ \\
\hline $\mathbf{F}_{\mathbf{1}}$ & 165 & 170 \\
$\mathbf{F}_{2}$ & 100 & 110 \\
$\mathbf{F}_{3}$ & 1560 & 1610 \\
\hline
\end{tabular}

Due to the implicit relationship between the structural parameters and the structural response (displacement), the Latin Hypercube Sampling method and Design of Experiment method are employed. The displacement of the frame is obtained by finite element method (FEM) in commercial software Abaqus. After that, the equation of $d\left(E, F_{1}, F_{2}, F_{3}\right)$ is obtained by Moving Least Square Response Surface Method, and we reach the limit-state function. At last the hybrid reliability is obtained as $95.85 \%$. The frame seems to be relatively reliable on its static state, but structural optimization should be performed when dynamic factors are considered.

\section{Conclusion}

In this paper, a hybrid reliability analysis method based on universal grey number is proposed. The method can calculate the reliability of structure under simultaneous random, interval, and fuzzy uncertainty. It can conclude a more accurate result, comparing with traditional interval analysis, because the universal grey number has the ability of reducing interval expansion. Moreover, the universal grey number calculation process is simple and has precise results. Three examples, including a engineering problem, demonstrate that our method can achieve more conservative outcomes compared with those obtained from interval analysis directly.

\section{Conflicts of Interest}

No potential conflicts of interest were reported by the authors.

\section{Acknowledgments}

This work was financially supported by the National Natural Science Foundation of China [Grant no. 11672104] and the National Science Fund for Distinguished Young Scholars in China [Grant no. 11225212].

\section{References}

[1] A. H.-S. Ang and W. H. Tang, Probability Concepts in Engineering Planning and Design. Volume II: Decision, Risk and Reliability, John Wiley \& Sons, New York, NY, USA, 1984.

[2] J. Liu, Q. Qing, Y. Deng, G. Wen, and H. Yin, "Fatigue reliability study on T-welded component considering load shedding," Fatigue \& Fracture of Engineering Materials \& Structures, vol. 38, no. 7, pp. 780-788, 2015.

[3] L. A. Zadeh, “Fuzzy sets," Information and Control, vol. 8, no. 3, pp. 338-353, 1965.

[4] J. Liu, G. Wen, and Y. M. Xie, "Layout optimization of continuum structures considering the probabilistic and fuzzy directional uncertainty of applied loads based on the cloud model,"
Structural and Multidisciplinary Optimization, vol. 53, no. 1, pp. 81-100, 2016.

[5] Y. Ben-Haim, "Convex models of uncertainty in radial pulse buckling of shells," Transactions of ASME: Journal of Applied Mechanics, vol. 60, no. 3, pp. 683-688, 1993.

[6] Y. Ben-Haim, "A non-probabilistic concept of reliability," Structural Safety, vol. 14, no. 4, pp. 227-245, 1994.

[7] I. Elishakoff, "Discussion on: a non-probabilistic concept of reliability," Structural Safety, vol. 17, no. 3, pp. 195-199, 1995.

[8] X. P. Du, "Interval reliability analysis," in International Design Engineering Technical Conferences Computers and Information in Engineering conference, The American Society of Mechanical Engineers, Cleveland, Ohio, USA, 2007.

[9] X. P. Du, A. Sudjianto, and B. Q. Huang, "Reliability-Based Design With the Mixture of Random and Interval Variables," in ASME 2003 Design Engineering Technical Conference and Computers and Information in Engineering Conference (DETC2003), American Society of Mechanical Engineers, Chicage, IL, USA, 2005.

[10] C. Jiang, W. X. Li, X. Han, L. X. Liu, and P. H. Le, "Structural reliability analysis based on random distributions with interval parameters," Computers \& Structures, vol. 89, no. 23, pp. 22922302, 2011.

[11] L. Wang, D. Liu, Y. Yang, X. Wang, and Z. Qiu, "A novel method of non-probabilistic reliability-based topology optimization corresponding to continuum structures with unknown but bounded uncertainties," Computer Methods Applied Mechanics and Engineering, vol. 326, pp. 573-595, 2017.

[12] Z. Qiu, D. Yang, and I. Elishakoff, "Probabilistic interval reliability of structural systems," International Journal of Solids and Structures, vol. 45, no. 10, pp. 2850-2860, 2008.

[13] J. Liu, G. Wen, Q. Qing, and Y. M. Xie, "An efficient method for topology optimization of continuum structures in the presence of uncertainty in loading direction," vol. 14, 2017.

[14] F. Li, G. Sun, X. Huang, J. Rong, and Q. Li, "Multiobjective robust optimization for crashworthiness design of foam filled thin-walled structures with random and interval uncertainties," Engineering Structures, vol. 88, pp. 111-124, 2015.

[15] L. Wang, X. Wang, Y. Li, G. Lin, and Z. Qiu, "Structural timedependent reliability assessment of the vibration active control system with unknown-but-bounded uncertainties," Structural Control and Health Monitoring, vol. 24, no. 10, 2017.

[16] W. Gao, D. Wu, K. Gao, X. Chen, and F. Tin-Loi, "Structural reliability analysis with imprecise random and interval fields," Applied Mathematical Modelling, vol. 55, pp. 49-67, 2018.

[17] Z. Meng, G. Li, D. Yang, and L. Zhan, "A new directional stability transformation method of chaos control for first order reliability analysis," Structural and Multidisciplinary Optimization, vol. 55, no. 2, pp. 601-612, 2017.

[18] L. Wang, X. Wang, H. Su, and G. Lin, "Reliability estimation of fatigue crack growth prediction via limited measured data," International Journal of Mechanical Sciences, vol. 121, pp. 44-57, 2017.

[19] J. Liu and G. Wen, "Continuum topology optimization considering uncertainties in load locations based on the cloud model," Engineering Optimization, vol. 50, no. 6, pp. 1041-1060, 2018.

[20] J. Liu, G. Wen, X. Chen, and Q. Qing, "Topology optimization of continuum structures with uncertainty in loading direction," in Proceedings of the 6th International Conference on Mechanics and Materials in Design, Ponta Delgada, Portugal, 2015. 
[21] A. Haldar and R. K. Reddy, "A random-fuzzy analysis of existing structures," Fuzzy sets and systems, vol. 48, no. 2, pp. 201-210, 1992.

[22] Z. Ni and Z. P. Qiu, "Hybrid probabilistic fuzzy and non-probabilistic model of structural reliability," Computers \& Industrial Engineering, vol. 58, no. 3, pp. 463-467, 2010.

[23] L. Wang, X. Wang, D. Wu, M. Xu, and Z. Qiu, "Structural optimization oriented time-dependent reliability methodology under static and dynamic uncertainties," Structural and Multidisciplinary Optimization, pp. 1-19.

[24] Z. Qin, "Random fuzzy mean-absolute deviation models for portfolio optimization problem with hybrid uncertainty," Applied Soft Computing, vol. 56, pp. 597-603, 2017.

[25] J. Wu, Z. Luo, H. Li, and N. Zhang, "Level-set topology optimization for mechanical metamaterials under hybrid uncertainties," Computer Methods Applied Mechanics and Engineering, vol. 319, pp. 414-441, 2017.

[26] D. Wu and W. Gao, "Hybrid uncertain static analysis with random and interval fields," Computer Methods Applied Mechanics and Engineering, vol. 315, pp. 222-246, 2017.

[27] W. Wang, H. Gao, C. Zhou, and Z. Zhang, "Reliability analysis of motion mechanism under three types of hybrid uncertainties," Mechanism and Machine Theory, vol. 121, pp. 769-784, 2018.

[28] L. Wang, X. J. Wang, and Y. Xia, "Hybrid reliability analysis of structures with multi-source uncertainties," Acta Mechanica, vol. 225, no. 2, pp. 413-430, 2014.

[29] S. S. Rao and L. Berke, "Analysis of uncertain structural systems using interval analysis," AIAA Journal, vol. 35, no. 4, pp. 727735, 1997.

[30] G. J. Shao and J. B. Su, "Interval finite element method and its application on anti-slide stability analysis," Applied Mathematics and Mechanics, vol. 28, no. 4, pp. 521-529, 2007.

[31] D. B. Miracle and O. N. Senkov, "A critical review of high entropy alloys and related concepts," Acta Materialia, vol. 122, pp. 448-511, 2017. 


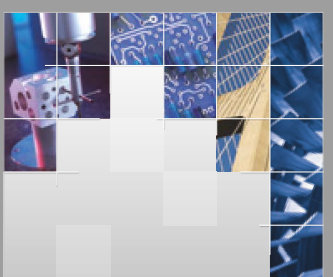

\section{Enfincering}
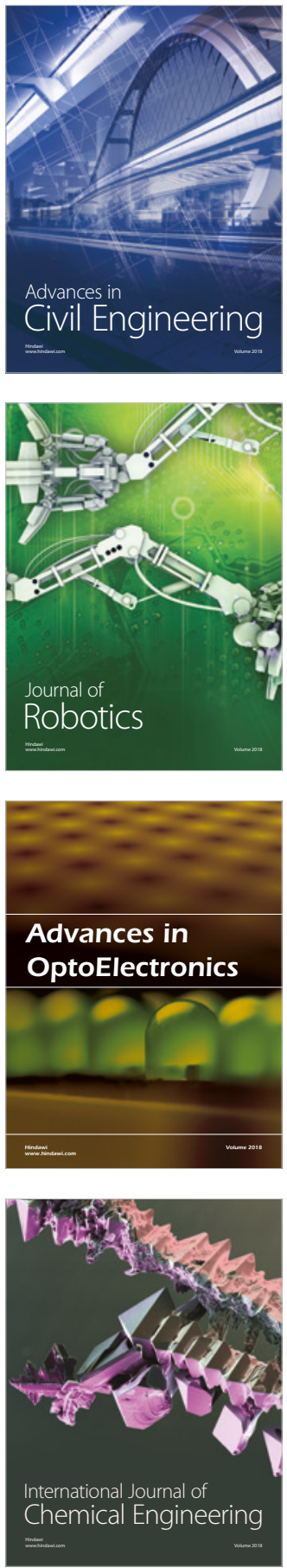

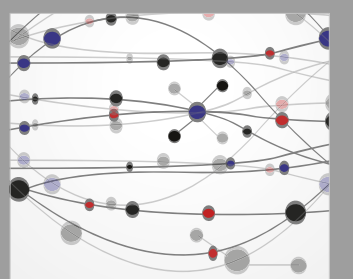

\section{Rotating \\ Machinery}

The Scientific World Journal

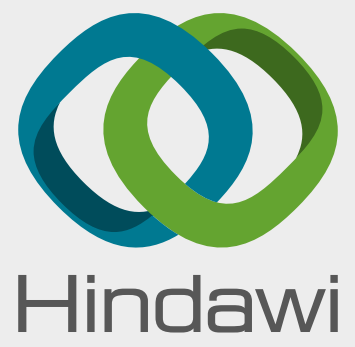

Submit your manuscripts at

www.hindawi.com
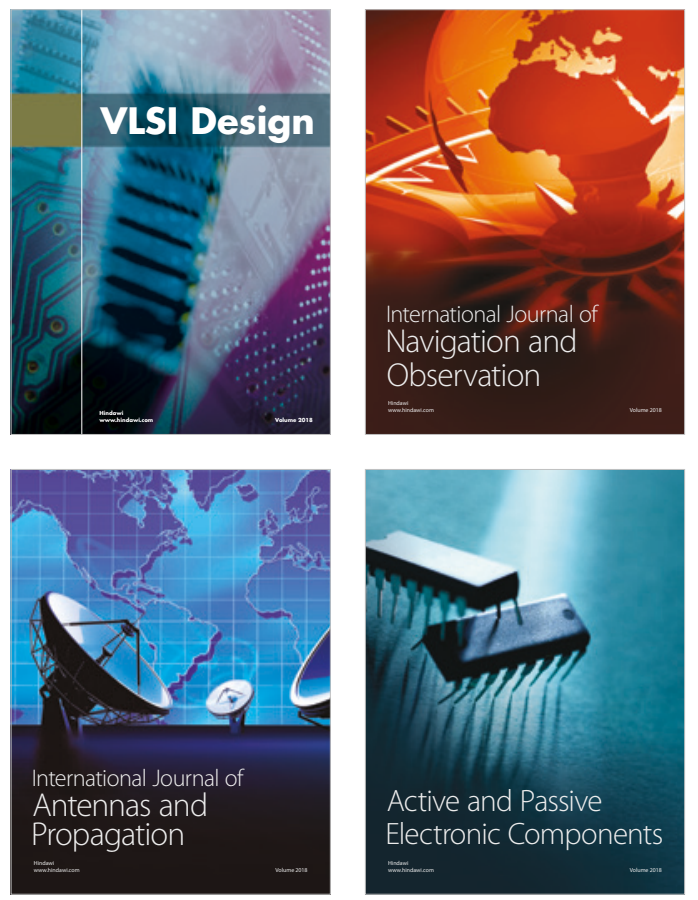
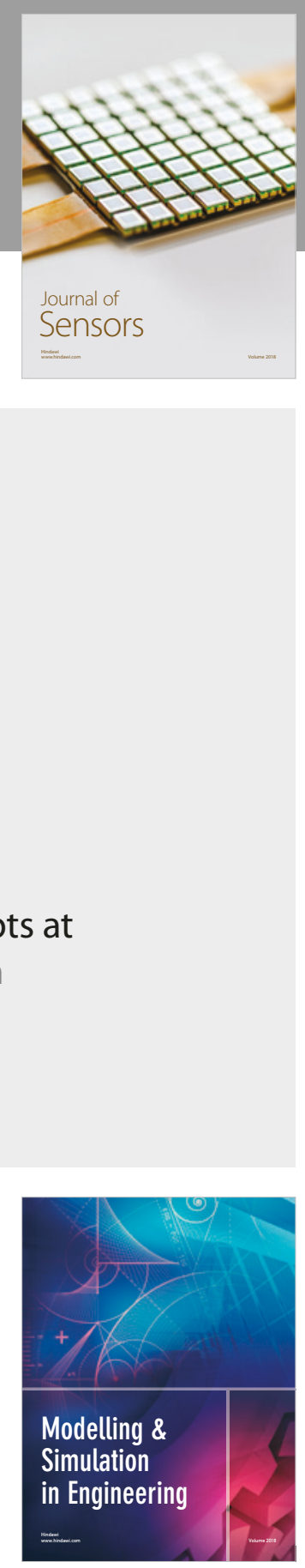

\section{Advances \\ Multimedia}
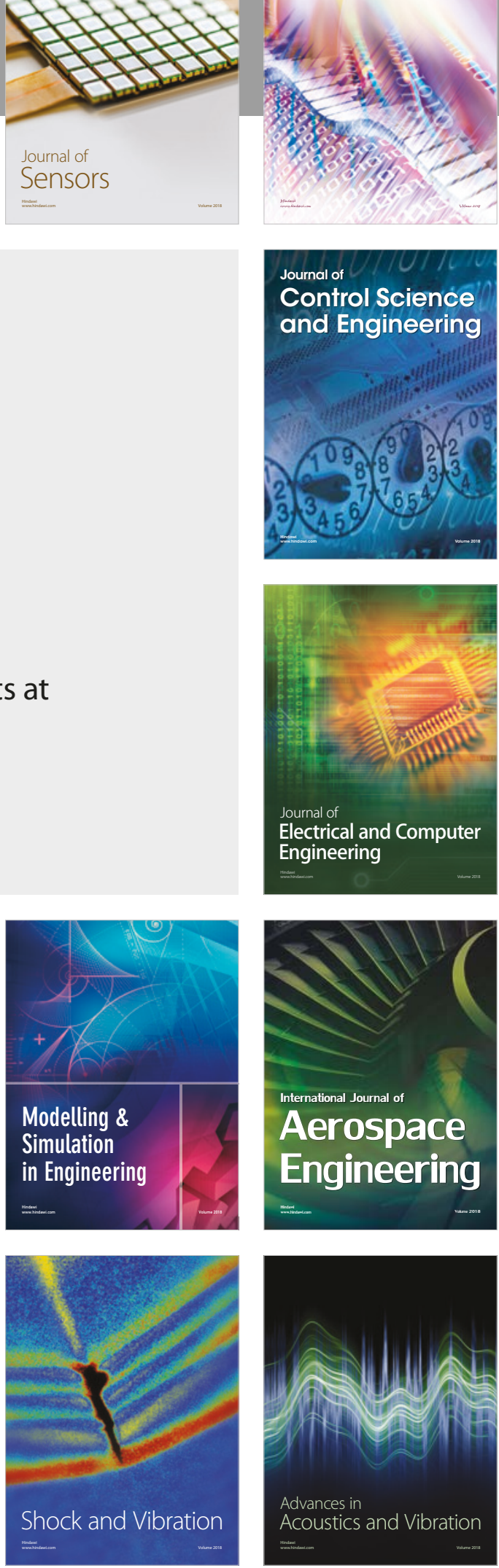